\title{
LacI-DNA-IPTG Loops: Equilibria among Conformations by Single-Molecule FRET
}

\author{
Zifan Wang, Kathy Goodson, Aaron R. Haeusler, Doug English, Jason D. Kahn \\ Wichita State University, Wichita, KS; University of Maryland, College Park, MD
}

\begin{abstract}
The E. coli Lac repressor (LacI) tetramer binds simultaneously to a promoter-proximal DNA binding site (operator) and an auxiliary operator, resulting in a DNA loop, which increases repression efficiency. Induction of the lac operon by allolactose reduces the affinity of LacI for DNA, but induction does not completely prevent looping in vivo. Single molecule fluorescence resonance energy transfer (SM-FRET) on a dual fluorophore-labeled LacI-9C14 loop showed that it adopts a single, stable, high-FRET V-shaped LacI conformation. Ligand-induced changes in loop geometry can affect loop stability. SM-FRET confirms that the high-FRET LacI-9C14 loop is only partially destabilized by saturating IPTG. FRET histograms suggest that the remaining population is a mixture of lower-FRET states ascribed to specificnonspecific or extended LacI loops, not free DNA.
\end{abstract}

\section{Introduction}

The control of the lac operon in E. coli by Lac repressor (LacI) is a classic example of negative gene regulation. The LacI homotetramer (a dimer of dimers) has the ability to bind two operator sites simultaneously, forming a DNA loop. In part, the biological function of looping is that binding of one dimer to its operator increases the effective local concentration of the other operator around the other dimer, leading to increased occupancy of the second operator.

Probing the role of inducers, anti-inducers, and other allosteric effectors is important in understanding the regulation of protein-DNA looped complexes. In the presence of the artificial inducer, isopropyl- $\beta$, Dthiogalactoside (IPTG), there is about a 1000-fold decrease in the affinity of LacI for the operator. One IPTG molecule binds to the core domain of one monomer of LacI. The reorientation of the core domain increases the distance between DNA binding headpieces in a dimer, destabilizing the strong interactions between the headpiece and the DNA operator (figure 1).

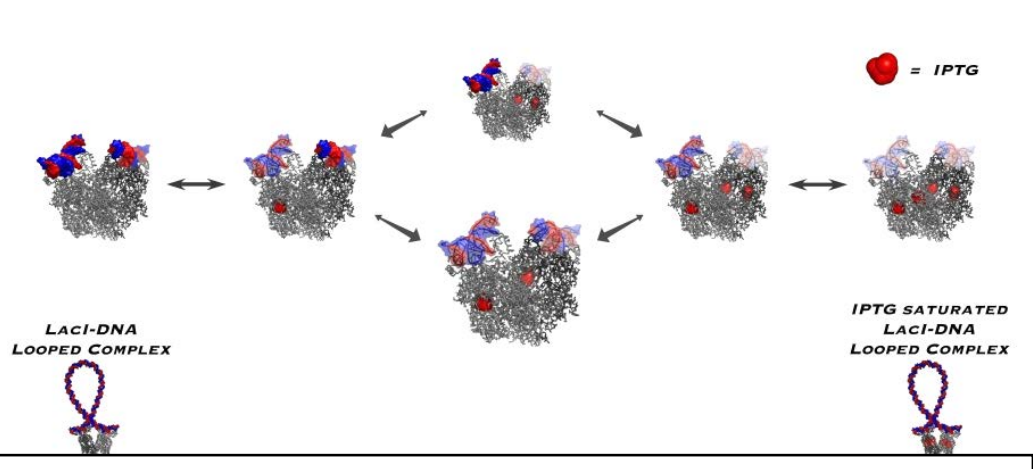

Figure1: One IPTG molecule binds to the core domain of one monomer of LacI with a $K_{D} \sim 5.0 \times 10^{-6} \mathrm{M}$
Our interest is in mutual interactions among inducer binding, DNA site selection, and DNA loop stability, and therefore we focus here on conditions where inducer is saturating, the DNA is designed to form exceptionally stable loops, and the protein:DNA ratio is $\sim 1$. A complete quantitative understanding of the lac operon, and by extension all genes regulated by ligand-responsive DNA looping proteins, will require confronting a more complete structural and thermodynamic characterization of all of the possible protein-DNAeffector complexes.

SM-FRET on freely diffusing LacI-DNA loops allows us to analyze population distributions directly as opposed to the average properties measured in a bulk experiment, and the efficiency of energy transfer should be highly sensitive to changes in loop geometry.

\section{Experiment, Results, Discussion, and Significance}

Fluorescently labeled 9C14: Fluorescently labeled oligonucleotides 56 nucleotides in length were used as PCR primers for synthesis of the 9C14 DNA with Alexa Fluor ${ }^{\circledR} 555$ (donor) and Alexa Fluor ${ }^{\circledR} 647$ (acceptor) fluorophores. Fluorophores were conjugated to oligonucleotides synthesized with an amino dT C-6 internal modification ( $\mathrm{T}^{*}$ below: iAmMC6T). Fluorescently labeled oligonucleotides were purified on a $12 \%$ 
polyacrylamide (40:1 (w/w) acrylamide: bis-acrylamide), $8 \mathrm{M}$ urea gel. The primers were excised, eluted, phenolchloroform extracted and ethanol precipitated. Dual fluorophore labeled DNA constructs were synthesized using the Phusion $^{\mathrm{TM}}$ High-Fidelity PCR System with the template described above. PCR reactions $(50 \mu \mathrm{L})$ contained 40 picograms of template, $200 \mu \mathrm{M}$ of each dNTP, $1 \mu \mathrm{M}$ each labeled primer, 1 X Phusion HF Reaction Buffer, and 2 units of Phusion ${ }^{\mathrm{TM}}$ High-Fidelity DNA Polymerase. PCR cycling conditions were the following: $94.0^{\circ} \mathrm{C}$ for $1 \mathrm{~min}$, $55.0^{\circ} \mathrm{C}$ for $30 \mathrm{~s}, 60.0^{\circ} \mathrm{C}$ for $30 \mathrm{~s}, 72^{\circ} \mathrm{C}$ for $1 \mathrm{~min}$, for 35 cycles. Purification was carried out as described for the radiolabeled DNA.

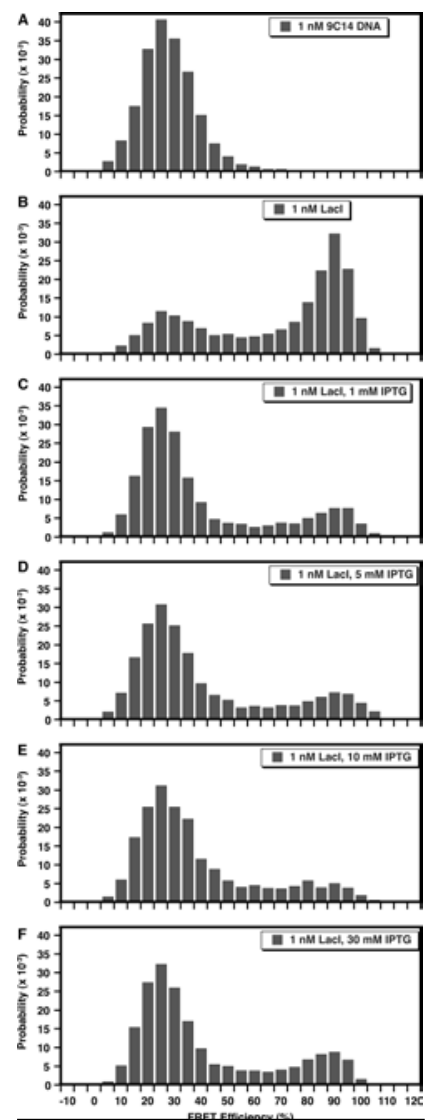

Figure2:Single-molecule fluorescence experiments on freely diffusing LacI-9C14IPTG complexes.
SM-FRET measurements: Single-molecule FRET was done essentially as described . Initial LacI titration experiments were used to determine the optimum concentration of LacI to be used. SM-FRET IPTG measurements were conducted at $1 \mathrm{nM} 9 \mathrm{C} 14$ and 1-1.5 nM LacI in LacI buffer. IPTG was added at various concentrations and stages as described in the text. Samples were allowed to equilibrate at room temperature $\left(20^{\circ} \mathrm{C}\right)$ for at least $5 \mathrm{~min}$. Single molecule studies were performed with an oil immersion objective inverted microscope (Fluar, 100X, N.A. $=1.3$, Carl Zeiss, Oberkochen, Germany) with standard methods. Excitation at $514 \mathrm{~nm}$ was from an argon ion laser focused $10 \mu \mathrm{m}$ into the sample from the glassliquid interface. The objective collects the fluorescent burst data as the molecules travel through the beam, and it is then directed through a notch filter (Kaiser Optical, Ann Arbor, MI) to remove the excitation wavelength of the laser. The emitted light is subsequently split between two avalanche photodiode single photon counting modules (SPCM-AQR-15, PerkinElmer Optoelectronics, Vaudreuil, Quebec) using a dichroic beam splitter (625DCLP, Chroma, Rockingham, VT). The photon counts are recorded on separate channels of a counter/timer board (PCI 6602, National Instruments, Austin, TX) in $1 \mathrm{~ms}$ time bins. The SPCMs are connected to the counter/timer board directed by Labview 6.0 (National Instruments) software. Results: We compare the FRET results on loop stability with an electrophoretic mobility shift assay (EMSA). Use SM-FRET experiments to investigate conformational changes caused by the addition of IPTG to pre-looped LacI-induced DNA complexes (figure 2).

\section{Conclusions}

SM-FRET, a convenient method to study DNA looping and binding.Effector molecules and architectural DNA binding proteins regulate loop shape and stability. Inducer bound Lac repressor undergoes a conformational change that allosterically affects the headpiece and core domains of the structure resulting in dramatically decreased affinity for DNA.The high local concentration of the operators allows the formation of LacI-DNA loops despite saturating [IPTG] in the supercoiled DNA environment.

\section{Acknowledgements}

This work was supported in part by a grant from the NSF and by the Wichita State University Foundation.

\section{Reference}

1. Jacob, F., and J. Monod. 1961. Genetic regulatory mechanisms in the synthesis of proteins. J. Mol. Biol. 3:318-356.

2. Friedman, A. M., T. O. Fischmann, and T. A. Steitz. 1995. Crystal structure of lac repressor core tetramer and its implications for DNA looping. Science 268:1721-1727.

3. Lewis, M., G. Chang, N. C. Horton, M. A. Kercher, H. C. Pace, M. A. Schumacher, R. G Brennan, and P. $\mathrm{Lu}$. 1996. Crystal structure of the lactose operon repressor and its complexes with DNA and inducer. Science 271:1247-1254.

4. Oehler, S., M. Amouyal, P. Kolkhof, B. von Wilcken-Bergmann, and B. Müller-Hill. 1994. Quality and position of the three lac operators of E. coli define efficiency of repression. EMBO J. 13:3348-3355.

5. Phillips, R., J. Kondev, and J. Theriot. 2008. Physical biology of the cell. Garland Science, New York. 